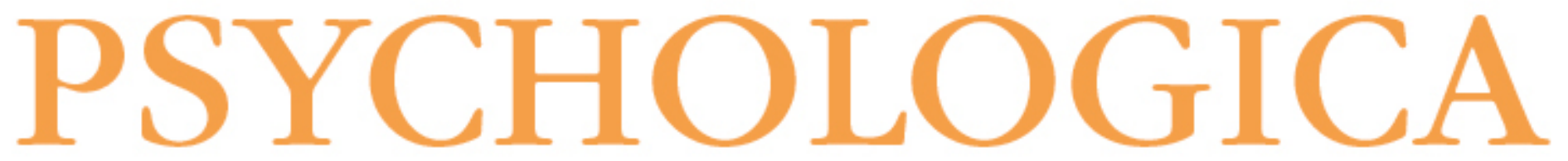

\title{
Questionário de Avaliação do Perfil Sazonal: características psicométricas da versão portuguesa do Seasonal Pattern Assessment Questionnaire (SPAQ)
}
Autor(es):
Fonte, Aníbal; Coutinho, Bruno
Publicado por: Imprensa da Universidade de Coimbra
URL
persistente:
URI:http://hdl.handle.net/10316.2/38640
DOI:
DOI:http://dx.doi.org/10.14195/1647-8606_58-1_5
Accessed : $\quad$ 26-Apr-2023 16:01:57

A navegação consulta e descarregamento dos títulos inseridos nas Bibliotecas Digitais UC Digitalis, UC Pombalina e UC Impactum, pressupõem a aceitação plena e sem reservas dos Termos e Condições de Uso destas Bibliotecas Digitais, disponíveis em https://digitalis.uc.pt/pt-pt/termos.

Conforme exposto nos referidos Termos e Condições de Uso, o descarregamento de títulos de acesso restrito requer uma licença válida de autorização devendo o utilizador aceder ao(s) documento(s) a partir de um endereço de IP da instituição detentora da supramencionada licença.

Ao utilizador é apenas permitido o descarregamento para uso pessoal, pelo que o emprego do(s) título(s) descarregado(s) para outro fim, designadamente comercial, carece de autorização do respetivo autor ou editor da obra.

Na medida em que todas as obras da UC Digitalis se encontram protegidas pelo Código do Direito de Autor e Direitos Conexos e demais legislação aplicável, toda a cópia, parcial ou total, deste documento, nos casos em que é legalmente admitida, deverá conter ou fazer-se acompanhar por este aviso.

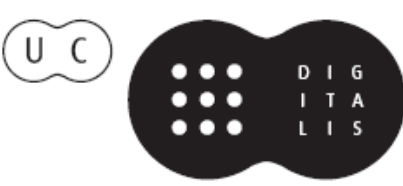




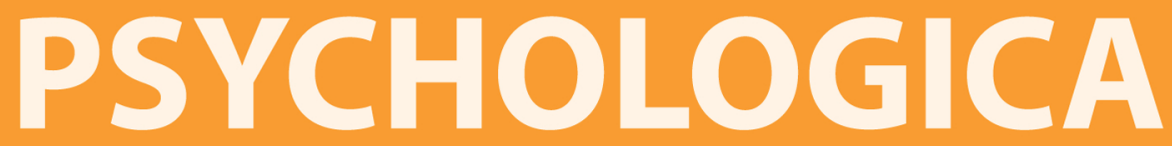

IMPRENSA DA UNIVERSIDADE DE COIMBRA COIMBRA UNIVERSITY PRESS

FACULDADE DE PSICOLOGIA E DE CIÊNCIAS

DA EDUCAÇÃO DA UNIVERSIDADE DE COIMBRA 


\title{
Questionário de Avaliação do Perfil Sazonal - Características psicométricas da versão portuguesa do Seasonal Pattern Assessment Questionnaire (SPAQ)
}

\author{
Aníbal Fonte ${ }^{1}$ e Bruno Coutinho ${ }^{2}$
}

Seasonal Pattern Assessment Questionnaire (SPAQ) - Psychometric characteristics of the Portuguese version

Abstract

Introduction/Objective: Seasonal alterations in mood and behavior characterized by depressive states during autumn/winter and remission during spring/summer are very frequent. In order to identify the sensitivity of the subjects to seasonal alterations, the Seasonal Pattern Assessment Questionnaire (SPAQ) is likely the most used self-evaluation instrument. The present study evaluates the psychometric properties of the Portuguese version of SPAQ.

Material and methods: The sample was composed of university and professional courses' students (137 men and 187 women; mean age $27.7 \pm 9.8$ ). The analysis of SPAQ focused on items that are part of to the Global Seasonality Score (GSS), and the categorization of subjects into subtypes: without seasonal affective disorder; subsyndromal seasonal affective disorder (S-SAD); seasonal affective disorder (SAD).

Results: The mean global seasonality score was $9.1 \pm 4.3$ showing no significant differences between men and women. The internal consistency of the items that constitute the Global Seasonality Score was 0.804 (Cronbach's $\alpha$ ) and the average inter-item correlation was

1 Departamento de Psiquiatria e Saúde Mental, Unidade Local de Saúde do Alto Minho. Hospital de Santa Luzia. Email: afonte@mail.telepac.pt

2 Departamento de Psiquiatria e Saúde Mental, Unidade Local de Saúde do Alto Minho. Hospital de Santa Luzia. Email: dr.couto@gmail.com. 
$\mathrm{r}=0.406$. In respect to the questionnaire's answers, $29.0 \%$ corresponds to subsyndromal seasonal affective disorder and $12.7 \%$ to seasonal affective disorder. Irrespective of the severity, $30.6 \%$ of the subjects considered seasonal alterations in mood and behavior a problem.

Conclusion: The SPAQ proved to be an easy-to-use self-evaluation instrument with good psychometric properties. Considering the sample size, the results suggest that the seasonal variations in humor and behavior are very common. The fact that higher values were found in comparison to other studies conducted in non-clinical populations highlights the necessity to evaluate the specificity of the questionnaire and conduct studies in representative population samples.

Keywords: seasonal affective disorder; Seasonal Pattern Assessment Questionnaire; seasonality; reliability; factor analysis

\section{Resumo}

Introdução/Objetivo: Alterações sazonais do humor e do comportamento caracterizadas por estados depressivos no outono/inverno e remissão na primavera/verão são condições muito frequentes. Para identificar a sensibilidade dos sujeitos às alterações sazonais o Seasonal Pattern Assessment Questionnaire (SPAQ) é provavelmente o instrumento de autoavaliação mais utilizado. O presente estudo avalia as propriedades psicométricas da versão portuguesa do $S P A Q$.

Material e métodos: A amostra foi constituída por estudantes universitários e de cursos profissionais (137 homens, 187 mulheres; média das idades $=27.7 \pm 9.8$ ). A análise do SPAQ centrou-se nos itens que constituem a Pontuação Global de Sazonalidade - PGS (Global Seasonality Score - GSS) e na categorização dos sujeitos em subtipos: sem perturbação afetiva sazonal (sem PAS); subsíndrome da perturbação afetiva sazonal (S-PAS); perturbação afetiva sazonal (PAS).

Resultados: A média da Pontuação Global de Sazonalidade foi de $9.1 \pm 4.3$, não se verificando diferenças significativas entre homens e mulheres. A consistência interna dos itens que constituem a Pontuação Global de Sazonalidade foi 0.804 ( $\alpha$ de Cronbach) e a correlação média inter-itens de $r=0.406$. Com base nas respostas ao questionário, $29.0 \%$ correspondiam a subsíndrome afetiva sazonal e $12.7 \%$ a perturbação afetiva sazonal. Independentemente da gravidade, $30.6 \%$ dos inquiridos consideravam as alterações sazonais do humor e do comportamento como problema.

Conclusão: O SPAQ revela ser um instrumento de autoavaliação com boas propriedades psicométricas, fácil de aplicar. Considerando a amostra estudada os resultados sugerem que as variações sazonais do humor e do comportamento são muito frequentes. $\mathrm{O}$ facto 
de se encontrarem valores superiores ao de outros estudos efetuados em populações não clínicas evidencia a necessidade de se avaliar a especificidade do questionário e de se realizarem estudos em amostras representativas da população.

Palavras-chave: perturbação afetiva sazonal; Seasonal Pattern Assessment Questionnaire; sazonalidade; fiabilidade; análise fatorial

Embora variações sazonais do humor e do comportamento sejam reconhecidas desde a antiguidade, a sua identificação como perturbação psiquiátrica apenas se desenvolveu a partir da década de 80 do século XX com os trabalhos realizados no National Institute of Mental Health (NIMH), em Bethesda, Maryland (EUA), por Norman Rosenthal e colaboradores. No desenrolar desses estudos, o termo Perturbação Afetiva Sazonal (PAS) foi adotado por Rosenthal et al., em 1984, para designar uma síndrome caracterizada por episódios depressivos recorrentes no outono ou inverno, com remissão na primavera ou verão, ocorrendo, pelo menos, dois anos consecutivos, não explicados por qualquer variação nos fatores psicossociais (Rosenthal et al., 1984).

Na mesma década, em 1987, a terceira edição revista do Diagnostic and Statistical Manual of Mental Disorders (DSM-III-R), editado pela American Psychiatric Association (1987), inscreve as manifestações sazonais do humor como "padrão sazonal" especificador de subgrupos de doentes com perturbações afetivas (perturbação bipolar I, perturbação bipolar II, ou perturbação depressiva major recorrente), definindo critérios de diagnóstico praticamente sobreponíveis aos de Rosenthal et al. (1984). Com algumas alterações nos critérios, sobretudo na determinação do número de dias para início e remissão dos sintomas, as edições posteriores do $D S M$, incluindo a mais recente ( $D S M-5)$, vão manter o "padrão sazonal" como especificador (American Psychiatric Association, 2013). Por sua vez, a Classificação Internacional de Doenças/Perturbações Mentais e do Comportamento, 10a ed. (ICD-10) (World Health Organization, 1992), não a reconhece diretamente remetendo-a para a perturbação depressiva recorrente ou para a perturbação bipolar, com o argumento de serem necessários mais estudos antes de ser internacionalmente aceite (WHO, 1993).

Apesar destas controvérsias, prosseguem os estudos sobre as hipóteses explicativas das variações sazonais do humor, abrangendo variação do fotoperíodo (Mersch, Middendorp, Bouhuys, Beersma \& Van den Hoofdakker, 1999b), dessincronização dos ritmos circadianos endógenos com o ciclo dia/noite (Lewy et al., 2007, 2009), alteração na secreção de melatonina (Arendt, 2003; Lam \& Levitan, 2000; Wehr et al., 2001), disfunção da síntese e turnover dos neurotransmissores e neuromo- 
deladores (Lambert, Reid, Kaye, Jennings \& Esler, 2002; Neumeister et al., 2001), fatores genéticos (Partonen et al., 2007; Sher, 2001).

Paralelamente têm sido conduzidos numerosos estudos epidemiológicos para investigar a prevalência da Perturbação Afetiva Sazonal (PAS). Winkler e Kasper (2005), num artigo em que reúnem vinte e dois estudos apontam para uma prevalência de PAS, nos países de clima temperado, entre $2 \%$ e $5 \%$. A prevalência nas mulheres tende a ser superior à dos homens, numa razão que varia entre 3.5:1 e 9:1 e, na maioria dos casos, o primeiro episódio de perturbação afetiva sazonal ocorre perto dos trinta anos. Todavia, entre os diversos estudos, verifica-se grande variabilidade nas taxas de prevalência para o que concorrem diferentes metodologias de avaliação e de investigação e especificidades das amostras como aclimatização ao meio e contextos socioculturais (Enns, Levitan, Levitt, Dalton \& Lam, 1999; Magnusson \& Partonen, 2005; Mersch et al., 1999b).

Quanto aos sintomas, a perturbação afetiva sazonal compartilha com a depressão major não sazonal os sintomas nucleares da depressão (humor deprimido, falta de energia, perda da capacidade de sentir prazer ou de interesse). Contudo, desde os trabalhos pioneiros de Rosenthal e colaboradores que se destaca, nesta perturbação, uma inversão no padrão dos sintomas vegetativos que a aproximam da depressão atípica: aumento da duração do sono, aumento do apetite com aumento de peso e alteração dos hábitos alimentares com ânsia por alimentos com hidratos de carbono (Enns et al., 1999; Magnusson \& Partonen, 2005). Quanto aos ciclos sazonais, embora a forma mais típica seja a que corresponde à depressão outono-inverno, nalgumas pessoas as manifestações surgem no verão com remissão dos sintomas depressivos no outono e inverno (Magnusson \& Partonen, 2005; Rastad, Sjödén \& Ulfberg, 2005; Wehr, Sack \& Rosenthal, 1987).

A intensidade com que os sintomas se podem apresentar é muito variável. Num dos extremos encontram-se variações sazonais sem sintomatologia clinicamente significativa, dentro dos padrões normais de variabilidade do humor e do comportamento e no outro extremo perturbações afetivas sazonais cujas manifestações podem corresponder à perturbação afetiva bipolar tipo II, com fases de hipomania na primavera ou verão, considerando-se muito raras as fases de mania (Magnusson \& Partonen, 2005; Rosenthal et al., 1984).

Para o estudo das perturbações afetivas sazonais na população tem-se recorrido a inquéritos e questionários de rastreio sendo provavelmente o Seasonal Pattern Assessment Questionnaire (SPAQ) (Rosenthal et al., 1984) o instrumento mais utilizado, independentemente da latitude e do país, com numerosas traduções em que se incluem versões em chinês (Han et al., 2000), japonês (Ozaki, Ono, Ito \& Rosenthal, 1995), tailandês (Srisurapanont \& Intaprasert, 1999), sami (lapónia) (Saarijarvi, Lauerma, Helenius \& Saarilehto, 1999), dinamarquês (Dam, Jakobsen \& Mellerup, 
1998), romeno (Soriano et al., 2007), italiano (Natale, Danesi \& Scapellato, 2003), holandês (Mersch, Middendorp, Bouhuys, Beersma \& Van den Hoofdakker, 1999a) e espanhol (Goikolea, Miralles, Bulbena Cabre, Vieta \& Bulbena, 2003).

Elaborado por forma a reconhecer a sensibilidade dos indivíduos às variações sazonais, num continuum e independente da presença de outras condições psicopatológicas, o SPAQ tanto pode ser utilizado em populações clínicas como não clínicas, tendo as suas características de fiabilidade, consistência interna, estabilidade temporal e de validade, sido demonstradas em diversos estudos (Magnusson, Friis \& Opjordsmoen, 1997; Magnusson \& Partonen, 2005; Mersch et al., 2004; Rohan \& Sigmon, 2000), o que não obsta ter sido objeto de críticas designadamente por conduzir a resultados que podem sobrestimar a prevalência de depressão sazonal (Hansen, Skre \& Lund, 2008).

Do exposto resulta, por um lado, a importância do estudo da influência dos ciclos sazonais no humor e no comportamento para melhor se compreender a dinâmica dos processos envolvidos e, por outro lado, da necessidade de instrumentos de medida que possibilitem a compreensão dos fatores que participam no seu desenvolvimento. Neste âmbito, constituindo o Seasonal Pattern Assessment Questionnaire (SPAQ) um método de avaliação standard, torna-se necessário testar e verificar as propriedades psicométricas da versão portuguesa deste questionário para se assegurar a sua adequabilidade, o que constitui o objetivo do presente trabalho.

\section{METODOLOGIA}

A amostra foi constituída por estudantes do ensino superior universitário provenientes de vários cursos da Universidade do Minho e da Universidade Fernando Pessoa (medicina, reabilitação, enfermagem, engenharia) e alunos inseridos em programas de formação profissional, por forma a reunir uma amostra heterogénea composta por indivíduos dos dois sexos, com maior dispersão etária e níveis de escolaridade.

Participaram 324 indivíduos (137 homens e 187 mulheres). A média de idades foi de 27.7 anos $(\mathrm{Dp}=9.84)$, não sendo estatisticamente significativa a diferença de idades entre homens (média $=27.8 ; \mathrm{Dp}=10.01$ ) e mulheres (média $=27.5$; $\mathrm{Dp}=9.74)$. A maioria dos inquiridos eram solteiros $(32.7 \%$ dos homens e $41.4 \%$ das mulheres) e a escolaridade média foi de 13.0 anos (12.9 nos homens e 13.1 nas mulheres) (Quadro 1). 
Quadro 1

Caracterização da Amostra

\begin{tabular}{rcccc}
\cline { 2 - 4 } & $\mathbf{H}+\mathbf{M}$ & Homens & Mulheres & \\
& $\mathbf{N}=\mathbf{3 2 4}$ & $\mathbf{N}=\mathbf{1 3 7}$ & $\mathbf{N}=\mathbf{1 8 7}$ & \\
\hline Idade. Média (Dp) & $27.7(9.84)$ & $27.8(10.01)$ & $27.5(9.74)$ & $t=0.260(\mathrm{gl}=322), \mathrm{NS}$
\end{tabular}

Estado civil. N. ${ }^{\circ}(\%)$

Solteiro

$240(74.1)$

$106(32.7)$

$134(41.4)$

Casado

$71(21.9)$

$26(8.0)$

$45(13.9)$

Div./Viúvo

$13(4.0)$

$5(1.5)$

$8(2.5)$

$\chi^{2}=2.016(\mathrm{gl}=3), \mathrm{NS}$

Escolaridade (anos)

Média (Dp) $13.0(3.18)$ $12.9(3.14)$ $13.1(3.22)$ $t=0.629(\mathrm{~g} \mid=322), \mathrm{NS}$

$t=\mathrm{t}$ Student; $\chi^{2}=$ qui quadrado; NS = não significativo

\section{SUJEITOS E MÉTODOS}

\section{Instrumentos}

Questionário de Avaliação do Perfil Sazonal (Seasonal Pattern Assessment Questionnaire - SPAQ). Instrumento desenvolvido por Rosenthal et al. (1984) para avaliação das alterações do humor e do comportamento ao longo do tempo, apresenta-se organizado em seis secções. A primeira seç̧ão compreende dez itens, pedindo-se ao sujeito que assinale o mês ou meses em que "se sente melhor", "tem tendência para ganhar peso", "relaciona-se mais socialmente", "dorme menos", "come mais", "perde mais peso", "relaciona-se menos socialmente", "sente-se pior", "come menos" ou "dorme mais", havendo ainda a possibilidade de assinalar para cada caso a resposta "não se destaca nenhum mês em particular".

A segunda secção é formada por seis itens (duração do sono, atividade social, humor, peso, apetite, nível de energia), devendo o sujeito indicar em cada um a suscetibilidade às mudanças segundo a estação do ano, numa escala de cinco pontos, desde "não muda" (0 pontos) a "muda muitíssimo" (4 pontos). A soma dos 
pontos nestes seis itens (total $=0$ a 24 pontos) constitui a Pontuação Global de Sazonalidade - PGS (Global Seasonality Score - GSS), correspondendo as pontuações mais elevadas a maior sensibilidade às mudanças sazonais.

$\mathrm{Na}$ terceira parte pede-se aos inquiridos para indicarem se as mudanças sazonais constituem um problema e, em caso afirmativo, qual a sua intensidade, de "leve" (1 ponto) a “incapacitante" (5 pontos).

Por último, nas secções quatro, cinco e seis, são registadas informações sobre alterações sazonais relacionadas com o peso corporal, horas de sono e preferências alimentares.

A conjugação da pontuação atribuída à Pontuação Global de Sazonalidade (PGS) com as respostas sobre perceção das mudanças sazonais como problema permitiu a Kasper, Wehr, Bartko, Gist \& Rosenthal (1989) estabelecer critérios para definir três grupos: sem perturbação afetiva sazonal (sem PAS), subsindrome da perturbação afetiva sazonal (S-PAS), perturbação afetiva sazonal (PAS). De acordo com esta tipologia, considera-se sem perturbação afetiva sazonal quando a Pontuação Global de Sazonalidade é $<9$ ou igual a 9 ou 10 e as alterações não constituem problema ou são leves (=1). Subsíndrome da perturbação afetiva sazonal (definida como um cluster de sintomas sazonais sem intensidade suficiente para permitir o diagnóstico de perturbação afetiva sazonal) é reconhecida quando PGS $=9$ ou 10 e as alterações sazonais são pontuadas como problema igual ou superior a moderado $(\geq 2)$, ou PGS $\geq 11$ e as alterações sazonais não constituem problema ou são leves $(=1)$. No terceiro grupo, classifica-se como perturbação afetiva sazonal quando PGS $\geq 11$ e as alterações sazonais são um problema pontuado, no mínimo, como moderado $(\geq 2)$. Esta combinação de pontuações é frequentemente utilizada em estudos epidemiológicos ainda que os critérios nem sempre sejam os mesmos, particularmente na delimitação entre S-PAS e PAS e na forma de valorizar a resposta à perceção das alterações sazonais como problema e respetivo nível de intensidade (Mersch et al., 1999b; Rastad et al., 2005).

No desenvolvimento do questionário para a versão em português além da tradução e retroversão para avaliação da correspondência semântica, lexical e cultural, procedeu-se a uma análise do conteúdo de cada item e à aplicação do questionário a um pequeno grupo de sujeitos semelhantes à população alvo constatando-se ser facilmente compreendido e respondido de acordo com as instruções.

\section{Procedimentos}

O procedimento iniciou-se com o pedido de autorização aos autores do questionário para a sua tradução e utilização. A aplicação do instrumento, acompanhado 
de uma folha para registo de dados sociodemográficos, ocorreu ao longo de 2011, com particular incidência nos meses de fevereiro a maio e outubro-novembro.

A análise estatística incidiu na avaliação da Pontuação Global de Sazonalidade (PGS) e nos critérios para a categorização em "sem PAS”, S-PAS e PAS. Além dos cálculos de estatística descritiva (média, mediana, moda), analisou-se a curva de distribuição de frequências através dos coeficientes de enviesamento (skewness) e de achatamento (kurtose) e utilizou-se o teste de Kolmogorov-Smirnov para examinar o ajustamento à distribuição normal. A análise de consistência interna da PGS foi efetuada através do coef. alpha de Cronbach e da correlação inter-item (r), considerando-se fraca uma correlação $<0.30$, moderada entre 0.30 e 0.70 e forte se $>0.70$ (Maroco \& Garcia-Marques, 2006). O teste $t$ de Student e a análise de variância (ANOVA two-way) foram utilizados, respetivamente, para a comparação das diferenças entre pontuações de duas amostras independentes e para amostras categorizadas a dois critérios. A associação entre variáveis ordinais foi avaliada através do coeficiente de Spearman por não exigir que os dados provenham de uma distribuição normal. Para o estudo das inter-relações entre os itens da PGS recorreu-se à análise dos componentes principais com rotação Varimax, determinando-se o número de fatores a reter a partir da observação do ponto de inflexão dos valores próprios (eigenvalues) no gráfico "scree plot" e para cada fator considerou-se como mínimo aceitável uma correlação de 0.40 entre as variáveis originais e as componentes principais.

O tratamento estatístico foi efetuado com recurso ao programa IBM SPSS Statistics 21.0 .

\section{Resultados}

O somatório dos seis itens da Pontuação Global de Sazonalidade apresentou a pontuação média de $9.06(\mathrm{Dp}=4.275)$, com um intervalo entre a pontuação mínima e máxima de $0-21$ (Quadro 2). As outras duas medidas de tendência central, mediana $=9$ e moda $=7$, situam-se igualmente à esquerda do ponto central da amplitude máxima desta variável (Fig. 1). Para melhor caracterizar a distribuição dos elementos da amostra, o recurso ao coeficiente de enviesamento (skewness $=-0.112)$ traduz a tendência para a distribuição ser assimétrica para a esquerda, enquanto o coeficiente de achatamento ( $k$ urtose $=-0.340$ ), também com valor negativo, retrata uma curva mais achatada do que a distribuição normal. $\mathrm{O}$ resultado do teste de Kolmogorov-Smirnov $(\mathrm{D}=0.076, \mathrm{p}<0.001)$ leva à rejeição da hipótese de normalidade o que reflete a distribuição assimétrica da pontuação dos elementos inquiridos. 
Quadro 2

Pontuação Global de Sazonalidade (PGS)

\begin{tabular}{lcccc}
\cline { 2 - 4 } & $\begin{array}{c}\mathbf{H}+\mathbf{M} \\
\mathbf{N}=\mathbf{3 2 4}\end{array}$ & $\begin{array}{c}\text { Homens } \\
\mathbf{N}=\mathbf{1 3 7}\end{array}$ & $\begin{array}{c}\text { Mulheres } \\
\mathbf{N}=\mathbf{1 8 7}\end{array}$ & \\
\hline PGS: Média (Dp) & $9.06(4.275)$ & $8.59(4.297)$ & $9.40(4.238)$ & $t=1.678(\mathrm{gl}=322), \mathrm{NS}$ \\
\multicolumn{1}{c}{ Limites } & $0-21$ & $0-21$ & $0-19$ & \\
PGS x Idade $^{*}$ & -0.094 & 0.102 & $-0.231^{* *}$ & \\
PGS x Escolaridade $^{*}$ & 0.074 & 0.019 & 0.069 & \\
\hline
\end{tabular}

${ }^{\star}$ Coeficiente de correlação de Spearman

** $\mathrm{P}<0.01$

Considerando a amostra dividida segundo o sexo (Quadro 2), os homens têm PGS média (8.59; $\mathrm{D} \mathrm{p}=4.297)$ inferior à das mulheres (9.40; $\mathrm{Dp}=4.238)$, porém a diferença não é estatisticamente significativa $(t=1.678, \mathrm{gl}=322$, NS). Quanto à idade e número de anos de escolaridade, quando os sujeitos da amostra são avaliados em conjunto não se verifica qualquer associação significativa com a Pontuação Global de Sazonalidade, mas se a amostra for dividida em homens e mulheres estas apresentam ligeira associação negativa e significativa entre a idade e PGS (Spearman R $=-0.231, \mathrm{p}<0.01$ ).

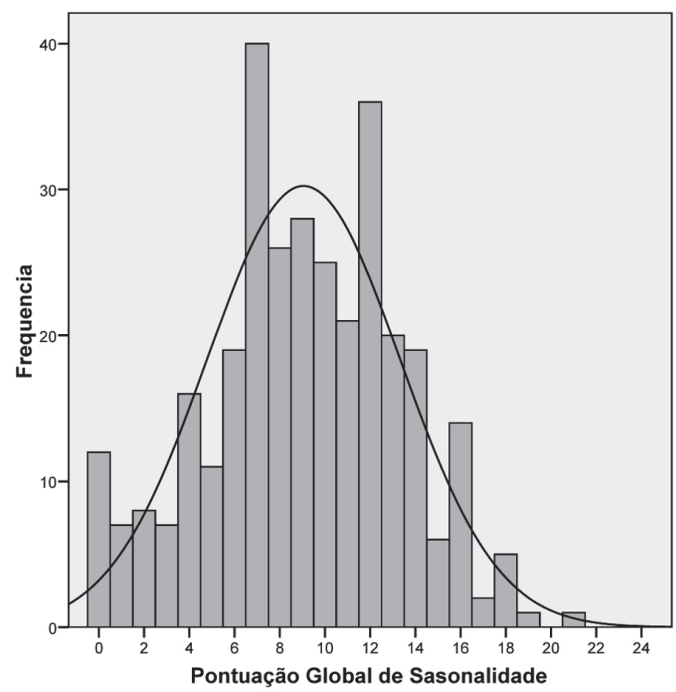

$\begin{aligned} \text { Mean } & =9,06 \\ \text { Std. Dev. } & =4,275 \\ N & =324\end{aligned}$

Figura 1. Distribuição de Frequências da Pontuação Global de Sazonalidade e Distribuição Ajustada pelo Teste de Kolmogorov-Smirnov. 
Relativamente à análise de consistência interna pelo coef. alpha de Cronbach, os seis itens da PGS têm bom nível de fiabilidade $(\alpha=0.804)$, com correlação média inter-itens moderada $(r=0.406)$.

No Quadro 3 apresenta-se, para cada item da PGS, os valores da pontuação média, desvio-padrão, correlação item-total e alpha de Cronbach se item eliminado. Os valores da média e desvio padrão de cada item mostram considerável homogeneidade e a correlação entre cada item e a pontuação total corrigida é, em geral, elevada, oscilando entre 0.393 e 0.656 , o que traduz congruência entre as respostas atribuídas a cada uma das questões que constituem a escala.

Quadro 3

Consistência Interna e Análise Fatorial da Pontuação Global de Sazonalidade

\begin{tabular}{lccccc}
\hline \multirow{2}{*}{ Item } & \multicolumn{3}{c}{ Análise de fiabilidade } & \multicolumn{2}{c}{ Análise fatorial ${ }^{* *}$} \\
\cline { 2 - 6 } & Média $(\mathbf{D p})$ & $\begin{array}{c}\text { Correlação } \\
\text { item-total }\end{array}$ & $\begin{array}{c}\text { a se item } \\
\text { eliminado }\end{array}$ & Fator 1 & Fator 2 \\
\hline Sono & $1.50(0.97)$ & 0.393 & 0.809 & $\mathbf{0 . 4 7 8}$ & 0.260 \\
Atividade social & $1.51(1,09)$ & 0.581 & 0.769 & $\mathbf{0 . 8 3 9}$ & 0.114 \\
Humor & $1.62(1.07)$ & 0.607 & 0.762 & $\mathbf{0 . 8 2 2}$ & 0.183 \\
Peso & $1.25(0.88)$ & 0.565 & 0.774 & 0.225 & $\mathbf{0 . 8 5 9}$ \\
Apetite & $1.35(0.99)$ & 0.574 & 0.770 & 0.239 & $\mathbf{0 . 8 7 4}$ \\
Nível de energia & $1.83(1.01)$ & 0.656 & 0.751 & $\mathbf{0 . 7 0 0}$ & 0.395 \\
\hline
\end{tabular}

${ }^{*} \mathrm{Na}$ pontuação total não está incluída o item em análise.

** Análise de componentes principais com rotação varimax. Para cada fator estão indicados a cheio e itálico os valores dos coeficientes ou pesos (loadings) $>0.40$.

A exploração da estrutura subjacente aos itens que compõem a escala foi efetuada através da análise de componentes principais com rotação ortogonal (varimax) determinando-se o número de fatores a extrair a partir do "scree plot" dos valores próprios (eigenvalues). Este procedimento levou a reter dois componentes principais que explicam 66.3\% da variância total (Quadro 3). O primeiro fator, que explica $51.0 \%$ da variância total, recolhe os itens "duração do sono", "atividade social”, "humor" e "nível de energia", enquanto o segundo fator, que explica $15.3 \%$ da variância total, agrupa as variações sazonais de "peso" e "apetite". Tanto o primeiro como o segundo fator apresentam boa consistência interna, respetivamente $\alpha=0.777$ e $\alpha=0.748$, e o coeficiente de correlação de Pearson entre os dois fatores é $\mathrm{r}=0.529(\mathrm{p}<0.001)$. 
Pontuação global de sazonalidade (PGS) e avaliação da gravidade

Na secção três do Questionário de Avaliação do Perfil Sazonal os sujeitos são solicitados a responder a duas questões: a) "as perturbações são um problema para si?"; b) se a resposta for afirmativa, qual o grau de gravidade (leve, moderado, grave, muito grave, incapacitante).

Quadro 4

Pontuação Global de Sazonalidade (PGS). Perceção das Alterações Sazonais como "Problema"

\begin{tabular}{|c|c|c|c|}
\hline & \multicolumn{2}{|c|}{ Problema } & \multirow{2}{*}{ Teste estatístico } \\
\hline & Não $(\mathrm{N}=225)$ & $\operatorname{Sim}(N=99)$ & \\
\hline \multicolumn{4}{|l|}{ PGS } \\
\hline Total: Média (Dp) & $8.38(4.135)$ & $10.59(4.214)$ & $t=4.393(\mathrm{gl}=322) \mathrm{p}<0.001$ \\
\hline \multicolumn{4}{|l|}{ Sexo: Média (Dp) } \\
\hline Homens & $7.96(4.016)$ & $10.74(4.590)$ & $t=3.280(\mathrm{gl}=135) \mathrm{p}<0.001$ \\
\hline \multirow[t]{2}{*}{ Mulheres } & $8.76(4.219)$ & $10.51(4.065)$ & $t=2.778(\mathrm{gl}=185) \mathrm{p}<0.01$ \\
\hline & & & $\mathrm{F}(1)=0.931 \mathrm{p}=0.335(\mathrm{NS})^{* *}$ \\
\hline \multicolumn{4}{|l|}{ Itens: Média (Dp) } \\
\hline Sono & $1.42(0.976)$ & $1.67(0.926)$ & $t=2.111(\mathrm{gl}=322) \mathrm{p}<0.05$ \\
\hline Atividade social & $1.42(1079)$ & $1.71(1.100)$ & $t=2.176(\mathrm{gl}=322) \mathrm{p}<0.05$ \\
\hline Humor & $1.45(1.052)$ & $2.00(1.020)$ & $t=4.349(\mathrm{gl}=322) \mathrm{p}<0.001$ \\
\hline Peso & $1.14(0.833)$ & $1.48(0.930)$ & $t=3.289(\mathrm{gl}=322) \mathrm{p}<0.01$ \\
\hline Apetite & $1.25(0.954)$ & $1.60(1.039)$ & $t=2.933(\mathrm{gl}=322) \mathrm{p}<0.01$ \\
\hline Nível de energia & $1.69(0.986)$ & $2.13(0.986)$ & $t=3.683(\mathrm{gl}=322) \mathrm{p}<0.001$ \\
\hline \multicolumn{4}{|l|}{ Gravidade do problema } \\
\hline \multicolumn{4}{|l|}{ Não $(n=225)$} \\
\hline Leve $(n=36)$ & & $8.75(4.404)$ & \\
\hline Moderado $(n=40)$ & & $11.08(3.058)$ & \\
\hline Grave $(n=18)$ & & $11.39(4.340)$ & \\
\hline Muito grave $(n=3)$ & & $16.67(1.155)$ & \\
\hline Incapacitante $(\mathrm{n}=2)$ & & $17.50(4.950)$ & \\
\hline
\end{tabular}

$t=$ teste $\mathrm{t}$ Student $\mathrm{F}=$ teste ANOVA; NS = não significativo

** ANOVA two-way (var. de medida: PGS; var. independentes: sexo, perceção do problema)

Neste estudo 99 sujeitos (30.6\%) responderam sim à primeira questão e, tanto em conjunto como separados segundo os sexos, os que responderam sim têm PGS média significativamente mais elevada do que os que responderam não (Quadro 4). 
Todavia, a diferença entre os valores médios não é afetada pelo fator sexo dos inquiridos, ou seja, o facto de ser homem ou mulher não influencia a resposta à perceção das alterações sazonais como "problema" e a pontuação global de sazonalidade, avaliada pela análise de variância (ANOVA) a dois fatores $(\mathrm{F}(1)=0.931 \mathrm{p}=0.335)$. Por sua vez, como se pode observar no Quadro 4, a perceção das alterações sazonais como problema reflete-se significativamente em todos os itens da subescala. Quanto à gravidade dos sintomas, a média da PGS progride desde o valor mais baixo nos que responderam "não" (média $=8.38$; $\mathrm{Dp}=4.135$ ) ou problema "leve" (média $=8.75 ; \mathrm{Dp}=4.404)$ até ao valor mais alto nos que responderam "incapacitante" (média $=17.50 ; \mathrm{Dp}=4.950)$ (Quadro 4).

Por último, no Quadro 5 apresenta-se a distribuição dos sujeitos da amostra segundo a PGS e a avaliação da gravidade do problema. De acordo com os critérios definidos por Kasper et al. (1989) no grupo de sujeitos com Perturbação Afetiva Sazonal (PAS) encontram-se 41 (12.7\%) elementos da amostra, 94 (29.0\%) correspondem ao Subsíndrome da Perturbação Afetivo Sazonal” (S-PAS) e 189 (58.3\%) revelam-se sem perturbação afetiva sazonal" (sem PAS).

Quadro 5

Pontuação Global de Sazonalidade (PGS) e Gravidade da Perturbação

\begin{tabular}{lccc}
\hline \multirow{2}{*}{ PGS (total) } & \multicolumn{3}{c}{ Pontuação global de sazonalidade } \\
\cline { 2 - 4 } & \multicolumn{1}{c}{} & $\mathbf{9}$ ou 10 & $\geq \mathbf{1 1}$ \\
\hline Sem problema & $115(35.5 \%)$ & $37(11.4 \%)$ & $73(22.5 \%)$ \\
Problema & & & \\
$\quad$ Leve & $19(5.9 \%)$ & $6(1.9 \%)$ & $11(3.4 \%)$ \\
Moderado & $7(2.2 \%)$ & $7(2.2 \%)$ & $26(8.0 \%)$ \\
Grave & $5(1.5 \%)$ & $3(0.9 \%)$ & $10(3.1 \%)$ \\
Muito grave & - & - & $3(0.9 \%)$ \\
Incapacitante & - & - & $2(0.6 \%)$ \\
\hline \multicolumn{1}{r}{ Total } & $146(45.1 \%)$ & $53(16.4 \%)$ & $125(38.6 \%)$ \\
\hline
\end{tabular}

\section{DISCUSSÃO}

O objetivo deste estudo foi avaliar as características psicométricas da versão portuguesa do SPAQ, pelo que apenas se teve em conta o grupo de questões relativas à variação dos sintomas ao longo das estações do ano (pontuação global de sazonalidade) sem se considerar a sua distribuição pelos 12 meses. 
Sendo a amostra inquirida procedente de populações não clínicas é compreensível que a pontuação global de sazonalidade se desloque para a pontuação mais baixa, traduzindo o facto de a maioria dos inquiridos não registar variações significativas do humor ou do comportamento segundo as estações do ano. Quanto à pontuação média da PGS é difícil estabelecer uma comparação com outros estudos dadas as diferentes características das populações inquiridas (idade, sexo, morbilidade, latitude, etc.), todavia, focando apenas estudos realizados em populações não clínicas nos países do sul da europa, os valores encontrados neste trabalho $(9.06 \pm 4.27)$ situam-se no intervalo dos resultados obtidos em Espanha $(8.09 \pm 4.15)($ Adan, Natale \& Fabbri, 2006) e Itália (9.71 \pm 3.95$)$ (Tonetti, Milfont, Tilyard \& Natale, 2013).

Relativamente à distribuição segundo o sexo, entre os homens e mulheres da amostra não se verificaram diferenças significativas na pontuação média da PGS, ao contrário do que sucedeu num estudo castelhano de características similares (Adan et al., 2006), ainda que neste último os sujeitos inquiridos fossem mais jovens (21.16 \pm 2.85 anos). Nesta área muitos estudos assinalam maior pontuação média da PGS nas mulheres do que nos homens (Dam et al., 1998; Rastad et al., 2005; Saarijarvi et al., 1999; Soriano et al., 2007; Tonetti et al., 2013), embora alguns trabalhos não registem essa diferença (Axelsson, Káradóttir \& Karlsson, 2002; Han et al., 2000). Quanto à associação entre PGS e idade regista-se na subamostra feminina uma ligeira, mas significativa, correlação negativa. $O$ facto de as mulheres e sujeitos mais jovens poderem ser mais sensíveis às variações sazonais é aflorado há mais de duas décadas (Dam et al., 1998; Mersch, Middendorp, Bouhuys, Beersma, \& Van den Hoofdakker, 1999a; Rastad et al., 2005; Saarijarvi et al., 1999) sem uma clara explicação (Axelsson et al., 2002), pelo que permanece como área a requerer mais investigação.

A avaliação da consistência interna dos seis itens da PGS apresenta valores elevados $(\alpha=0.804)$, em correspondência com outros estudos sobre as propriedades psicométricas do SPAQ (Adan et al., 2006; Magnusson et al., 1997; Mersch et al., 2004; Natale et al., 2003; Rastad et al., 2005) o que traduz a boa capacidade dos seis itens para avaliarem o constructo que lhe está subjacente (Maroco \& Garcia-Marques 2006). No que concerne à estrutura fatorial, o presente trabalho replica os de Adan et al. (2006), Magnusson et al. (1997), Mersch et al. (2004) e Natale et al. (2003) com a definição de dois fatores principais responsáveis por $66.3 \%$ da variância total. Destes, o primeiro fator, que Mersch et al. (2004) caracteriza como "fator psicológico" (sono, atividade social, humor, energia) explica 51.0\% da variância, representando a máxima variação dos dados iniciais, enquanto o segundo fator ou "fator metabólico" (peso, apetite) ("food factor" na designação de Mersch et al., 2004) explica $15.3 \%$ da variância. Todavia, o facto de o segundo fator apre- 
sentar um valor próprio (eigenvalues) relativamente pequeno $(<1)$ e de os itens que o compõem registarem uma correlação com o total da PGS semelhante aos itens que integram o primeiro fator, secundando o raciocínio apresentado por Adan et al. (2006) e Magnusson et al. (1997), pode-se aceitar a unidimensionalidade da PGS, em que cada item avalia diferentes aspetos de um mesmo constructo.

Quanto aos sujeitos que consideram as manifestações sazonais como "problema", independentemente da avaliação da sua gravidade, neste estudo 30.6\% dos inquiridos inscrevem-se nesse grupo. Este valor aproxima-se dos $27.0 \%$ obtidos por Kasper et al. (1989) no estado de Maryland, EUA, através de inquérito por telefone, mas é muito inferior aos $81.7 \%$ registados num estudo efetuado entre estudantes, na China (Rastad et al., 2005). Na análise da resposta às variações sazonais do humor e do comportamento como "problema", além de fatores relacionados com vulnerabilidades biológicas e psicológicas, Mersch et al. (1999b) postulam a necessidade de se considerar a influência de diferenças culturais na aceitação em se admitir dificuldades psicológicos como um "problema”. Outra questão é a possibilidade de o SPAQ avaliar mais a sensibilidade à sazonalidade do que a perturbação afetiva sazonal (Mersch et al., 2004).

Neste estudo, a distribuição dos sujeitos pelas categorias definidas por Kasper et al. (1989) registou 12.7\% com perturbação afetiva sazonal e 29.0\% com subsíndrome afetivo sazonal. Estudos epidemiológicos baseados no SPAQ referem para a população geral taxas de prevalência de PAS entre 0.7\% e 12.4\% (Adan et al., 2006; Axelsson et al., 2002; Magnusson \& Partonen, 2005; Rastad et al., 2005) e de 2.6\% a 19.1\% para o S-PAS (Adan et al., 2006; Rastad et., 2005). Apesar de serem intervalos muito amplos, os valores obtidos situam-se acima dos limites superiores indicados. O SPAQ não diagnostica a perturbação afetiva sazonal per si, as respostas ao questionário apenas conduzem a uma estimativa baseada na autoavaliação retrospetiva da variação sazonal a um conjunto de seis sintomas. Confrontado com critérios mais restritivos (por exemplo, os definidos nas sucessivas revisões da DSM) é atualmente aceite que o SPAQ tende a sobrestimar os casos de perturbação afetiva sazonal (Blazer, Kessler \& Swartz 1998; Magnusson \& Partonen, 2005; Mersch et al., 1999b; Thompson, Thompson, \& Smith, 2004) e tanto a especificidade como o seu valor preditivo têm sido objeto de críticas (Raheja, King \& Thompson, 1996; Thompson \& Cowan, 2001).

Dois instrumentos têm sido apresentados em alternativa ao SPAQ: i) "Structured Interview Guide for the Hamilton Depression Rating Scale - Seasonal Affective Disorders Version Self-Rating Format" (SIGH-SAD-SR). Inventário de autoavaliação, derivado de uma entrevista estruturada (Williams, Link, \& Terman, 1994), com duas subescalas, sendo uma de 21 itens da Escala de Depressão de Hamilton e a segunda de oito itens para sintomas atípicos; dada a sua extensão é geralmente utilizada 
como complemento do SPAQ para avaliação dos potenciais casos de PAS e análises de tratamentos (Flory, Ametepe \& Bowers, 2012; Rastad, Ulfberg \& Lindberg, 2011). ii) "Seasonal Health Questionnaire (SHQ)", questionário de autoavaliação, apontado como tendo maior especificidade do que o SPAQ (Thompson \& Cowan, 2001; Thompson, Thompson, \& Smith, 2004), é mais longo e difícil de preencher e requer nas primeiras perguntas que a pessoa tenha sofrido de depressão major nos últimos anos para que possa continuar a responder.

Conceptualmente distinto, o SPAQ baseia-se na presença de seis sintomas atribuídos aos estados depressivos (duração do sono, atividade social, humor, peso, apetite, nível de energia) e na perceção que o próprio tem das suas alterações sazonais, devendo-se notar que no presente estudo não se procedeu à discriminação dos estados depressivos segundo os meses do ano nem se teve em conta "janelas temporais" para validar as alterações sazonais, o que poderia conduzir à exclusão de alguns casos tidos como perturbação afetiva sazonal ou subsíndrome da perturbação afetiva sazonal e, em consequência, a um número menor de casos.

A finalidade deste trabalho foi de analisar a fiabilidade do questionário e estabelecer as suas propriedades psicométricas, inquirindo-se amostras de conveniência formadas por estudantes em diversos graus de ensino, podendo-se admitir enviesamentos face a amostras aleatórias. Os estudantes universitários, além das deslocações das suas residências habituais no final do verão e início do outono, estão sujeitos a ritmos sociais marcados pelos períodos de trabalho e de férias que podem influenciar o humor. Quanto aos inquiridos a frequentar cursos profissionais, o retorno aos estudos, muitas vezes após anos de interrupção, ocorria, maioritariamente, por estarem desempregados, situação que pode ter influenciado a seleção da amostra com incremento de elementos mais vulneráveis a PAS (Mersch et al., 1999a, 1999b).

Em síntese, os itens que constituem a escala da Pontuação Global de Sazonalidade do SPAQ revelam determinar de forma coerente e sinérgica um mesmo constructo, reunindo num cluster sintomas que tendem a ocorrer em conjunto. Considerado na sua globalidade, o SPAQ mostra ser um instrumento simples, fácil de utilizar e com boa fiabilidade.

Quanto à determinação da prevalência na população geral, os dados apontam para elevada frequência de variações sazonais do humor e do comportamento. Contudo, os resultados devem ser interpretados com prudência, sendo necessário prosseguir com estudos que: a) Avaliem as alterações sazonais do humor e do comportamento independentemente da apreciação subjetiva, retrospetiva e reportada a um longo período de tempo, realizada pelos próprios; b) Determinem a percentagem dos sujeitos com variações sazonais do humor que correspondem a situações clinicamente significativas; c) Estudem eventuais especificidades relacionadas 
com a latitude e o clima nas diferentes regiões de Portugal, comparando-as com as obtidas noutras zonas do globo; d) Analisem a especificidade do questionário SPAQ, comparando-o com o resultado de avaliações clínicas.

Ainda que a CID-10 e a DSM-5 não reconheçam a perturbação afetiva sazonal como entidade clínica mas apenas como especificação de subgrupos da depressão major e da perturbação bipolar, as suas repercussões no bem-estar e no desempenho familiar, profissional e social são frequentes e significativas para que possam ser ignoradas. O uso de um instrumento que possibilite a realização de rastreios e a determinação dos sujeitos com variações sazonais de humor revela-se tanto mais importante quanto se trata de uma perturbação subdiagnosticada que pode ser tratada com adequadas medidas terapêuticas.

\section{REFERÊNCIAS}

Adan, A., Natale, V., \& Fabbri, M. (2006). Propiedades psicométricas de la versión castellana del cuestionario de evaluación de patrón estacional (Seasonal Pattern Assessment Questionnaire, SPAQ). Revista Latinoamericana de Psicología, 38(1), 59-69.

American Psychiatric Association (1987). Diagnostic and statistical manual of mental disorders ( $3^{\text {rd }}$ ed., revised). Washington, DC: American Psychiatric Publishing.

American Psychiatric Association (2013). Diagnostic and statistical manual of mental disorders - DSM-5 ( $5^{\text {th }}$ ed.). Washington, DC: American Psychiatric Publishing.

Arendt J. (2003). Importance and relevance of melatonin to human biological rhythms. Journal of Neuroendocrinology, 15, 427-431.

Axelsson, J., Káradóttir, R., \& Karlsson, M. M. (2002). Differences in prevalence of seasonal affective disorder that are not explained by either genetic or latitude differences. International Journal of Circumpolar Health, 61(1), 17-20.

Blazer, D. G., Kessler, R. C., \& Swartz M. S. (1998). Epidemiology of recurrent major and minor depression with a seasonal pattern: The National Comorbidity Survey. British Journal of Psychiatry, 172, 164-167.

Dam, H., Jakobsen, K., \& Mellerup, E. (1998). Prevalence of winter depression in Denmark. Acta Psychiatrica Scandinavica, 97(1), 1-4.

Enns, M. W., Levitan, R. D., Levitt, A. J., Dalton, E. J., \& Lam, R. W. (1999). Diagnosis, epidemiology, and pathophysiology. In R. W. Lam \& A. J. Levitt (Eds.), Canadian consensus guidelines for the treatment of seasonal affective disorder (pp. 20-63). Vancouver (BC): Clinical \& Academic Publishing.

Flory, R., Ametepe, J., \& Bowers, B. (2012). A randomized, placebo-controlled trial of bright light and high-density negative air ions for treatment of Seasonal Affective Disorder. Psychiatry Research, 177, 101-108.

Goikolea, J., Miralles, G., Bulbena Cabre, A., Vieta, E., \& Bulbena, A. (2003). Adaptación española del cuestionario de evaluación de perfil estacional (Seasonal Pattern Assessment Questionnaire, SPAQ) en las versiones de adultos e infanto-juvenil. Actas Españolas de Psiquiatría, 3, 192-198. 
Han, L., Wang, K., Cheng, Y., Du, Z., Rosenthal, N. E., \& Primeau, F. (2000). Seasonal variations in mood and behavior among Chinese medical students. American Journal of Psychiatry, 157(1), 133-135.

Hansen, V., Skre, I., \& Lund, E. (2008). What is this thing called "SAD"? A critique of the concept of Seasonal Affective Disorder. Epidemiologia e Psichiatria Sociale, 17(2), 120-127.

Kasper, S., Wehr, T. A., Bartko, J., Gist, P., \& Rosenthal, N. E. (1989). Epidemiological findings of seasonal changes in mood and behavior: A telephone survey of Montgomery County, Maryland. Archives of General Psychiatry, 46, 823-833.

Lam, R. W., \& Levitan, R. D. (2000). Pathophysiology of seasonal affective disorder: A review. Journal of Psychiatry and Neuroscience, 25(5), 469-480.

Lambert, G. W., Reid, C., Kaye, D. M., Jennings, G. L., \& Esler, M. D. (2002). Effects of sunlight and season on serotonin turnover in the brain. The Lancet, 360(Dec.), 1840-1842.

Lewy, A. J., Emens, J. S., Songer, J. B., Sims, N., Laurie, A. L., Fiala, S. C., \& Buti, A. L. (2009). Winter Depression: Integrating mood, circadian rhythms, and the sleep/wake and light/dark cycles into a bio-psycho-social-environmental model. Sleep Medicine Clinics, 4(2), 285-299.

Lewy, A. J., Rough, J. N., Songer, J. B., Mishra, N., Yuhas, K., \& Emens, J. S. (2007). The phase shift hypothesis for the circadian component of winter depression. Dialogues in Clinical Neuroscience, 9(3), 291-300.

Magnusson, A., Friis, S., \& Opjordsmoen, S. (1997). Internal consistency of the seasonal pattern assessment questionnaire (SPAQ). Journal of Affective Disorders, 42(2-3), 113-116.

Magnusson, A., \& Partonen, T. (2005). The diagnosis, symptomatology, and epidemiology of seasonal affective disorder. CNS Spectrums, 10(8), 625-634.

Maroco, J., \& Garcia-Marques, T. (2006). Qual a fiabilidade do alfa de Cronbach? Questões antigas e soluções modernas? Laboratório de Psicologia, 4(1), 65-90.

Mersch, P., Middendorp, H., Bouhuys, A. L., Beersma, D., \& Van den Hoofdakker, R. H. (1999a). The prevalence of seasonal affective disorder in The Netherlands: A prospective and retrospective study of seasonal mood variation in the general population. Biological Psychiatry, 45(8), 1013-1022.

Mersch, P., Middendorp, H., Bouhuys, A. L., Beersma, D., \& Van den Hoofdakker, R. H. (1999b). Seasonal affective disorder and latitude: A review of the literature. Journal of Affective Disorders, 53(1), 35-48.

Mersch, P., Vastenburg, N. C., Meesters, Y., Bouhuys, A. L., Beersma, D. G., Van den Hoofdakker, R. H., \& Den Boer, J. A. (2004). The reliability and validity of the Seasonal Pattern Assessment Questionnaire: A comparison between patient groups. Journal of Affective Disorders, 80(2), 209-219.

Natale, V., Danesi, E., \& Scapellato, P. (2003). Validazione della versione Italiana del Seasonal Pattern Assessment Questionnaire (SPAQ). Testing Psicometria Metodologia, 10(1-2), 33-42.

Neumeister, A., Willeit, M., Praschak-Rieder, N., Asenbaum, S., Stastny, J., Hilger, E., Pirker, W., Konstantinidis, A., \& Kasper, S. (2001). Dopamine transporter availability in symptomatic depressed patients with seasonal affective disorder and healthy controls. Psychological Medicine, 31(08), 1467-1473.

Ozaki, N., Ono, Y., Ito, A., \& Rosenthal, N. E. (1995). Prevalence of seasonal difficulties in mood and behavior among Japanese civil servants. American Journal of Psychiatry, 152, 1225-1227.

Partonen, T., Treutlein, J., Alpman, A., Frank, J., Johansson, C., Depner, M., ... Schumann, G. (2007). Three circadian clock genes Per2, Arnlt and Npas2 contribute to winter depression. Annals of Medicine, 39(3), 229-238. 
Raheja, S. K., King, E. A., \& Thompson, C. (1996). The Seasonal Pattern Assessment Questionnaire for identifying seasonal affective disorders. Journal of Affective Disorders, 41, 193-199.

Rastad, C., Sjödén, P. O., \& Ulfberg, J. (2005). High prevalence of self-reported winter depression in a Swedish county. Psychiatry and Clinical Neurosciences, 59(6), 666-675.

Rastad, C., Ulfberg, J., Lindberg, P. (2011). Improvement in fatigue, sleepiness, and health-related quality of life with bright light treatment in persons with Seasonal Affective Disorder and Subsyndromal SAD. Depression Research and Treatment, 2011. doi: http://dx.doi.org/10.1155/2011/543906

Rohan, K. J, \& Sigmon, S. T. (2000). Seasonal mood patterns in a northeastern college sample. Journal of Affective Disorders, 59, 85-96.

Rosenthal, N. E., Sack, D. A., Gillin, J. C., Lewy, A. J., Goodwin, F. K., Davenport, Y., Mueller, P. S., Newsome, D. A., \& Wehr, T. A. (1984). Seasonal affective disorder. A description of the syndrome and preliminary findings with light therapy. Archives of General Psychiatry, 41(1), 72-80.

Saarijarvi, S., Lauerma, H., Helenius, H., \& Saarilehto, S. (1999). Seasonal affective disorders among rural Finns and Lapps. Acta Psychiatrica Scandinavica, 99(2), 95-101.

Sher, L., (2001). Genetic studies of seasonal affective disorder and seasonality. Comprehensive Psychiatry, 42(2), 105-110.

Soriano, J. J., Ciupagea, C., Rohan, K. J., Neculai, D. B., Yousufi, S. M., Guzman, A., \& Postolache, T. T. (2007). Seasonal variations in mood and behavior in Romanian postgraduate students. The Scientific World Journal, 7, 870-879.

Srisurapanont, M, \& Intaprasert, S. (1999). Seasonal variations in mood and behaviour: Epidemiological findings in the north tropics. Journal of Affective Disorders, 54, 97-99.

Thompson, C., Cowan, A. (2001). The seasonal health questionnaire: A preliminary validation of a new instrument to screen for seasonal affective disorder. Journal of Affective Disorders, 64, 89-98.

Thompson, C., Thompson, S., Smith, R. (2004). Prevalence of seasonal affective disorder in primary care: A comparison of the seasonal health questionnaire and the seasonal pattern assessment questionnaire. Journal of Affective Disorders, 78, 219-226.

Tonetti, L., Milfont, T. L., Tilyard, B. A., \& Natale, V. (2013). Month of birth and mood seasonality: A comparison between countries in the northern and southern hemispheres. Psychiatry and Clinical Neurosciences, 67(3), 133-138.

Wehr, T. A., Duncan, W. C., Sher, L., Aeschbach, D., Schwartz, P. J., Turner, E. H., Postolache, T. T., \& Rosenthal, N. E. (2001). A circadian signal of change of season in patients with seasonal affective disorder. Archives of General Psychiatry, 58(12), 1108-1114.

Wehr, T. A., Sack, D. A., \& Rosenthal, N. E. (1987). Seasonal affective disorder with summer depression and winter hypomania. American Journal of Psychiatry, 144, 1602-1603.

Williams, J. B. W., Link, M. J., \& Terman, M. (1994). Instructions for the SIGH-SAD-SR, Structured Interview Guide for the Hamilton Depression Rating Scale-Seasonal Affective Disorder Version, Self-Rating Version. New York State Psychiatric Institute and Columbia University, New York, NY, USA.

Winkler, D., \& Kasper, S. (2005). Seasonal affective disorder: From diagnosis to treatment. Medicographia, 27, 247-253.

World Health Organization (1992). The ICD-10 Classification of Mental and Behavioural Disorders: Clinical descriptions and diagnostic guidelines. WHO, Geneva.

World Health Organization (1993). The ICD-10 Classification of Mental and Behavioural Disorders: Diagnostic criteria for research. WHO, Geneva. 